\title{
Analysis of BNIP3 and BNIP3L/Nix expression in cybrid cell lines harboring two LHON-associated mutations*
}

\author{
Agata Kodron ${ }^{\natural}$, Parvana Hajieva², Agata Kulicka1', Bohdan Paterczyk², Elona Jankauskaite ${ }^{1}$ \\ and Ewa Bartnik ${ }^{1,4} \otimes$ \\ IInstitute of Genetics and Biotechnology, Faculty of Biology, University of Warsaw, Warsaw, Poland; 2Institute of Pathobiochemistry, University \\ Medical Center of the Johannes Gutenberg University, Mainz, Germany; ${ }^{3}$ Laboratory of Electron and Confocal Microscopy, Faculty of Biology, \\ University of Warsaw, Warsaw, Poland; ${ }^{4}$ nstitute of Biochemistry and Biophysics, Polish Academy of Sciences, Warsaw, Poland
}

\begin{abstract}
Mitochondria are key players in cell death through the activation of the intrinsic apoptosis pathway. BNIP3 and BNIP3L/Nix are outer mitochondrial membrane bifunctional proteins which because they contain both $\mathrm{BH} 3$ and LIR domains play a role in the cellular response to stress by regulation of apoptosis and selective autophagy. Leber's Hereditary Optic Neuropathy (LHON) is the most common mitochondrial disease in adults, characterized by painless loss of vision caused by atrophy of the optic nerve. The disease in over $90 \%$ of cases is caused by one of three mutations in the mitochondrial genome: $11778 \mathrm{G}>\mathrm{A}, 3460 \mathrm{G}>\mathrm{A}$ or $14484 \mathrm{~T}>\mathrm{C}$. The pathogenic processes leading to optic nerve degeneration are largely unknown, however, the most common explanation is that mtDNA mutations increase the apoptosis level in this tissue. Here we present the results of analysis of BNIP3 and BNIP3L/Nix proteins in cells harboring a combination of the 11778G $>$ A and the 3460G $>$ A LHON mutations. Experiments performed on cybrids showed that the BNIP3 protein level is decreased in LHON cells compared to controls. CCCP treatment resulted in apoptosis induction only in control cells. Moreover, we also noticed a reduced level of autophagy in LHON cybrids. The presented results suggest that in cells carrying LHON mutations expression of BNIP3 proteins involved in regulation of apoptosis and autophagy is decreased which in turn may disturb cell death pathways in those cells and affect cellular response to stress.
\end{abstract}

Key words: mitochondria, BNIP3, BNIP3L, LHON, apoptosis, autophagy

Received: 11 June, 2019; revised: 20 August, 2019; accepted: 16 September, 2019; available on-line: 04 October, 2019

区e-mail: agatakodron@wp.pl (AK); ewambartnik@gmail.com (EB) *Acknowledgements of Financial Support: This work was sup ported by grants 2014/15/B/NZ2/02272 and 2011/03/N/NZ3/00655 from the National Science Centre. Experiments were carried out with the use of CePT infrastructure financed by the European Union - the European Regional Development Fund (Innovative economy 2017-2013, Agreement POIG.02.02.00-14-024/08-00).

Abbreviations: OXPHOS, Oxidative Phosphorylation; mtDNA, mitochondrial DNA; RGCs, Retinal Ganglion Cells; LHON, Leber's hereditary optic neuropathy; BNIP3, Bcl-2/adenovirus E1B $19 \mathrm{kDa}-$ interacting protein 3; BNIP3L/Nix, $\mathrm{BCl}-2$ interacting protein 3 like; LC3, Microtubule-associated protein 1A/1B-light chain 3; LIR, LC3 interacting region; $\mathrm{BH}, \mathrm{BCl}-2$ homology; $\mathrm{ROS}$, Reactive Oxygen Species; CCCP, carbonylcyanide-3-chlorophenylhydrazone; MALM, Mieap-induced accumulation of lysosome-like organelles within mitochondria

\section{INTRODUCTION}

Mitochondria are organelles which generate ATP for the cell via oxidative phosphorylation (OXPHOS), a pathway consisting of five multisubunit enzyme complexes located within the mitochondrial inner membrane. Mitochondria contain their own circular DNA $(\mathrm{mtD}$ NA). The human mtDNA is about $16569 \mathrm{bp}$ long and encodes 13 polypeptides including seven subunits (ND1, ND2, ND3, ND4, ND4L, ND5, and ND6) of complex I, one subunit (cytochrome b) of complex III, three subunits (COI, COII, and COIII) of complex IV, and two subunits (ATPases 6 and 8) of complex V. Each mitochondrion has several mtDNA molecules, and each cell contains from several hundred to several thousand mitochondria. The existence of multiple DNA molecules, all equally susceptible to mutation, can lead to the phenomenon called "heteroplasmy", when two or more different mtDNA molecules are present in one cell (mutated and not mutated) (Stewart et al., 2015).

Apoptosis is a genetically regulated highly coordinated process of selective elimination of dysfunctional cells. Mitochondria play a key role in cell death regulation by activation of the intrinsic pathway of apoptosis in which several different proteins are involved. BNIP3 and BNIP3-like (BNIP3L or Nix, sharing 56\% homology with $\mathrm{BNIP} 3$ ) are $\mathrm{BH} 3$-only members of the $\mathrm{Bcl}-2$ family localized at the outer mitochondrial membrane which exert pro-apoptotic activity. BNIP3 and BNIP3L/Nix normally are present as monomers and upon activation in response to stress undergo homodimerization, bind to antiapoptotic proteins, like $\mathrm{Bcl}-2$ and $\mathrm{Bcl}-\mathrm{X}_{\mathrm{L}}$, and activate apoptosis effectors of Bax and Bak which permeabilize the mitochondrial outer membrane leading to release of cytochrome c, which in turn activates caspase-dependent cell death (Gustafsson et al., 2011; Hamacher-Brady et al., 2016). Moreover, through permeabilized transition pores, apoptosis inducing factor (AIF) and endonuclease $G$ are also released which are translocated to the nucleus and induce caspase-independent apoptosis (Liu et al., 2016). Autophagy is an evolutionarily conserved process of lysosome-mediated degradation of damaged cellular contents, including organelles, through structures called autophagosomes. Selective degradation of mitochondria can occur via two different pathways - PINK1/parkin dependent and through phosphorylation-regulated mitophagic receptors. The ubiquitin-marked mitochondrial proteins are recognized by p62 proteins which connect to LC3 proteins associated with the autophagosome membrane. In the next stage mitochondria are encapsulated in autophagosomes, where they are degraded after 
fusion with lysosomes. Receptor-mediated autophagy is based on activation of different outer membrane proteins which contain the LC3 interacting region (LIR) and directly bind to autophagosomal LC3 (LC3-II isoform). BNIP3 and Nix contain the LIR motif, therefore apart from involvement in apoptosis, they are also important regulators of mitochondrial autophagy (mitophagy) (Gustafsson et al., 2011; Imazu et al., 1999).

Mitochondrial diseases are a heterogeneous group of multi-system disorders, which may result from mutations either in nuclear genes or in mitochondrial DNA. A consequence of random distribution of $\mathrm{mtDNA}$ is differences in mutation load between tissues in the same individual or between individuals in the same family. In mitochondrial diseases multiple organs are affected, and the symptoms can occur either in infancy or during adulthood. However, the predominance of muscle and nervous tissue involvement is highly significant (Alston et al., 2017). Fibroblasts and transmitochondrial cytoplasmic hybrids, called cybrids, are the cell models most commonly used in mitochondrial studies. Cybrids are derived from the fusion of cells deprived of mitochondrial DNA (called $\varrho 0$ cells) by long culture on ethidium bromide and cytoplasts, enucleated cells containing the analyzed mitochondria originating from the patient's cells. The resulting cells have nuclei from $\varrho 0$ cells, but mitochondria from the patient, and this allows the analysis and comparison of the effect of different mitochondrial DNA variants in the same nuclear background Jankauskaite et al., 2017).

In 1988, Leber's hereditary optic neuropathy (LHON) was the first human pathology to be associated with a mitochondrial DNA point mutation (Mroczek-Tońska et al., 2003). To date, $35 \mathrm{mtDNA}$ mutations have been associated with LHON (according to MITOMAP, www. mitomap.org). They all are missense mutations, and most are located in Complex I genes. Currently, the three most frequent pathogenic mutations, called "primary mutations" (11778G >A/ND4, 3460G $>$ A/ND1, 14484T >C/ ND6) all affecting components of complex $\mathrm{I}$, are found in the majority of LHON patients. The clinical phenotype of LHON is the degeneration of retinal ganglion cells (RGCs) and a progressive degeneration of the optic nerve. In contrast to the pleiotropic phenotypes observed in other mitochondrial diseases, in most LHON patients the only symptom is vision loss. Occasionally, LHON is associated with neurological, cardiac, and skeletal symptoms. LHON has a markedly reduced penetrance with a clear sex bias. Approximately $50 \%$ of men and approximately $10 \%$ of women harboring one of the three primary pathogenic mutations develop visual failure. The age of onset is usually between 18 and 30 years, but an individual can become affected at any age between early childhood and over 70 years (Mroczek-Tońska et al., 2003). The reasons for the specificity of neurodegeneration in LHON are not clear. There are hypotheses that LHON mutations cause bioenergetic defects in cells, that LHON mitochondria produce more reactive oxygen species (ROS) or that the optic neurons are more sensitive to ROS. One of the most common explanations is that mtDNA mutations increase the level of apoptosis in this tissue (Mroczek-Tońska et al., 2003). Moreover, other types of cell death could also be involved in retinal ganglion cell degeneration in LHON, for example autophagy. The pathogenic processes leading to optic nerve atrophy are largely unknown, however, recent studies show that the treatment with a compound called idebenone can partially compensate for the deleterious effect of the 11778G $>$ A mutation in some cell lines (Yu-Wai-Man et al., 2017).

Programmed cell death is an essential process which maintains cellular homeostasis, therefore any perturbations in regulation and in the balance of proteins involved in apoptosis or autophagy lead to biochemical dysfunction. There are studies on the involvement of BNIP3 and BNIP3L/Nix in neurodegenerative diseases and cancer, but the contribution of those pro-apoptotic proteins to the pathogenesis of mitochondrial diseases is not known and their expression in cells with mtDNA mutations was not investigated. The aim of the present study was the analysis of BNIP3 and BNIP3L/Nix levels in a cell culture model expressing mutations responsible for LHON. The second goal was to study the correlation between BNIP3 and BNIP3L/Nix expression and apoptotic and autophagic cell death.

\section{MATERIALS AND METHODS}

Probands. In 2005, the three brothers were referred to the Department of Ophthalmology, Hospital of the Medical University of Warsaw with a suspicion of LHON. Patient III-1 at the age of 27 began to complain of deterioration of vision, first in the left then in right eye. In patient III-3 sudden loss of vision occurred before the age of 26 (exact age unknown). The loss of sight was also sequential, first in one and then in the second eye. In patient III-6 sudden loss of vision occurred at the age of 20. Fundus examination of three brothers showed pallor of the optic disc, narrowing of blood vessels and degeneration of the optic nerve. The patients' mother (II-1) and their sister (III-5) showed normal visual acuity and had no signs of degeneration of the optic nerve. All three men were smokers (Tońska et al., 2008).

In 2008 two other men from the same family (III-2 and III-7) were examined in the same hospital. Patient III-2 began to lose vision in the right eye at the age of 20 and the symptoms occurred in the second eye six months later. Patient III-7 began to lose his sight three months before he was taken to the hospital (at the age of 36) and loss of vision in this patient was first in the right eye, and after two months, decreased vision in the left eye was diagnosed. No data were available on the diagnostic characteristics of patient III-4, it is only known that he started to lose sight at the age of 25. None of the patients showed any additional neurological disorders besides blindness. Genetic studies conducted on DNA isolated from blood cells revealed in all symptomatic men, the asymptomatic sister and their mother the presence of two mutations responsible for the occurrence of LHON: 11778G $>\mathrm{A}$ and 3460G $>\mathrm{A}$ (Tońska et al., 2008). For the presented project material derived from 4 members of this family was taken. The double mutation $11778 \mathrm{G}>\mathrm{A}$ and $3460 \mathrm{G}>\mathrm{A}$ family pedigree is shown in Fig. 1. The study was approved by the Independent Ethics Committee of the Medical University of Warsaw (KB/183/2012).

Fibroblast cultures. Cultured fibroblasts from patients were derived from skin biopsies of four patients carrying the $11778 \mathrm{G}>\mathrm{A}$ and the $3460 \mathrm{G}>\mathrm{A}$ LHON mutations. Three age-matched control fibroblast cell lines (without mtDNA mutations) were derived by Dr. Aleksandra Sołyga-Żurek from patients of the Maria Skłodowska-Curie Memorial Cancer Centre and Institute of Oncology in Warsaw. Fibroblasts from patients and controls were cultured in complete DMEM medium 


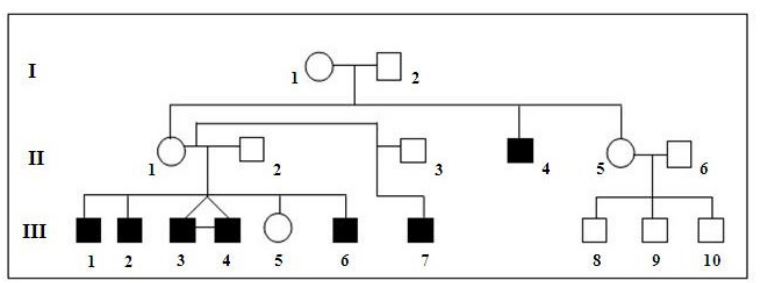

Figure 1 Pedigree of a Polish LHON family with the $11778 \mathrm{G}>\mathrm{A}$ and the $3460 \mathrm{G}>\mathrm{A}$ mutations.

Affected individuals are marked by filled squares. Four members of family were included in the studies. The probands were: III-3, III-4, III-6 and III-7 (Tońska et al., 2008).

(4.5 g/1 glucose; Life Technologies) supplemented with $10 \%$ (vol/vol) fetal bovine serum (Life Technologies), $1 \%$ (vol/vol) penicillin/streptomycin (Sigma), $1 \mathrm{mM}$ pyruvate (Life technologies) and $0.05 \mathrm{mg} / \mathrm{ml}$ uridine (Sig$\mathrm{ma})$.

Construction of cybrid cell lines. Cybrid cell lines were constructed using enucleated fibroblasts derived from skin biopsies of 4 male patients with double LHON mutations and 3 healthy controls. Fibroblasts were enucleated by twenty-four hours of incubation of $0.3 \times 10^{6}$ cells in complete medium supplemented with actinomycin D (Sigma) (final concentration $2 \mu \mathrm{g} \mathrm{ml}$ ). Then enucleated cells were fused with $1.5 \times 10^{6}$ of Q0 143B osteosarcoma cells in the presence of polyethylene glycol (PEG; Sigma) ( $\varrho 0$ 143B cells were kindly provided by Dr. Łukasz Borowski). After $1 \mathrm{~min}$ of incubation PEG was washed out and cells were grown for twenty-four hours in complete DMEM medium. Twenty-four hours after fusion, complete medium was replaced by selective medium without pyruvate and uridine and supplemented with 5-bromo-2'-deoxyuridine (final concentration $0.1 \mathrm{mg} / \mathrm{ml}$ ). Cells were cultured in selective medium for 14 days, and after this time it was replaced by complete DMEM medium (4.5 g/l glucose; Life Technologies) supplemented with $10 \%$ (vol/vol) fetal bovine serum (Life Technologies), 1\% (vol/vol) penicillin/streptomycin (Sigma), $1 \mathrm{mM}$ pyruvate (Life Technologies) and $0.05 \mathrm{mg} / \mathrm{ml}$ uridine (Sigma). Cell lines used in the study were checked for contamination with mycoplasma using EZ-PCR ${ }^{\mathrm{TM}}$ Mycoplasma Test Kit (BI Biological industries).

Measurement of heteroplasmy and analysis of nuclear background of cybrids. Measurement of heteroplasmy level. Total genomic DNA was isolated from 4 LHON cybrid cell lines using a standard phenol/chloroform method, according to the procedure described by Tońska and others (Tońska et al., 2008). Heteroplasmy was determined by last-cycle hot PCR-RFLP. DNA amplification was performed by using reverse primer 5'-TGGGGAGGGGGGT'TCATAGTA-3' and forward primer 5'-CAGTCAGAGGTTCAATTCCTC-3' for the $11778 \mathrm{G}>\mathrm{A}$ mutation and reverse primer 5'-GCGAGGT'TAGCGAGGCTTGC-3' and forward primer 5'-CAGCCACATAGCCCTCGTAG-3' for the 3460G $>$ A mutation. PCR conditions were $94^{\circ} \mathrm{C}$ for $5 \mathrm{~min}, 35$ cycles with denaturation at $95^{\circ} \mathrm{C}$ for $30 \mathrm{~s}$, annealing at $58^{\circ} \mathrm{C}$ for $30 \mathrm{~s}$ and elongation at $72^{\circ} \mathrm{C}$ for $45 \mathrm{~s}, 1$ cycle at $72^{\circ} \mathrm{C}$ for $7 \mathrm{~min}$ and final hold at $4^{\circ} \mathrm{C}$. PCR products were radiolabeled with $\alpha-{ }^{32} \mathrm{P}-\mathrm{dATP}$ in an additional PCR cycle. The $230 \mathrm{bp}$ product for the $11778 \mathrm{G}>\mathrm{A}$ mutation was digested with MaeIII (Roche) and the $302 \mathrm{bp}$ product for the $3460 \mathrm{G}>\mathrm{A}$ mutation with BsaHI (New England Biolabs) and separated on a $12 \%$ nondenaturing polyacrylamide gel. The sequence containing the $11778 \mathrm{G}>\mathrm{A}$ transition was cleaved into two bands of $146 \mathrm{bp}$ and $84 \mathrm{bp}$ (wild type sequence was not cleaved) and the sequence containing the $3460 \mathrm{G}>\mathrm{A}$ mutation was not cleaved (wild type sequence was cleaved into two bands of $183 \mathrm{bp}$ and 119 bp). The radioactivity in each fragment was quantified using MultiGauge V3.0 software.

Nuclear background analysis. Total genomic DNA isolated from all analyzed cell lines (4 LHON and 3 controls) were checked for the common nuclear background of $\varrho 0143 \mathrm{~B}$ cells by analysis of 3 STR nuclear markers (THO1, CSF1PO and D21S11). In the first step of analysis 3 STR markers were amplified by the multiplex PCR method with starters labeled with 2 different fluorescent dyes (Supplementary Material Table A at https://ojs.ptbioch.edu.pl/index.php/abp/). PCR conditions were $95^{\circ} \mathrm{C}$ for $12 \mathrm{~min}, 35$ cycles with denaturation at $95^{\circ} \mathrm{C}$ for $30 \mathrm{~s}$, annealing at $60^{\circ} \mathrm{C}$ for $90 \mathrm{~s}$ and elongation at $72^{\circ} \mathrm{C}$ for $45 \mathrm{~s}, 1$ cycle at $72^{\circ} \mathrm{C}$ for $30 \mathrm{~min}$ and final hold at $4^{\circ} \mathrm{C}$. Following PCR amplification samples were sequenced directly on the ABI3730xl DNA Analyzer (Applied Biosystems) in the Laboratory of DNA Sequencing and Oligonucleotide Synthesis of the Institute of Biochemistry and Biophysics, Polish Academy of Sciences. The length of the obtained products was determined in the Peak Scanner 1.0 software and compared to the length of each allele for STR markers described in Short Tandem Repeat DNA Internet DataBase, what allowed to determine the alleles for each STR marker in the samples.

Apoptosis. Apoptosis was analyzed in cells cultured in complete medium and after treatment of cells with $10 \mu \mathrm{M}$ CCCP for $4 \mathrm{~h}$. The analysis of apoptosis in cell lines was carried out by measuring free nucleosome production. For each measurement $5 \times 10^{4}$ cells were used. The number of apoptotic cells was estimated using of the Cell Death Detection ELISA ${ }^{\text {Plus immunoenzymatic }}$ assay (Roche) according to the manufacturer's instructions. The absorbance at $405 \mathrm{~nm}$ was measured with the Paradigm Detection Platform (Beckman Coulter) plate reader. The level of absorbance was proportional to the number of free nucleosomes. The results were normalized to control cells.

Mitochondrial mass. Mitochondrial mass was measured in standard culture conditions using MitoTracker Green FM probes (Thermo Fisher Scientific). Mito'Tracker Green localizes to mitochondria regardless of mitochondrial membrane potential. Cells were plated on 24 -well plates at a density of $2 \times 10^{5}$ cells. Cells were incubated with pre-warmed medium containing $200 \mathrm{nM}$ MitoTracker dye at room temperature for 20 minutes, followed by two washing steps. Fluorescence was excited by wavelength $485 \mathrm{~nm}$ and emitted wavelength at $535 \mathrm{~nm}$ was measured in a Paradigm Detection Platform (Beckman Coulter) plate reader. The results were normalized to control cells. The mitochondrial mass was also checked by western blotting with the use of TOMM20 primary antibodies (Thermo Fisher Scientific).

Western blot analysis. Proteins used for analyses were isolated from cells cultured either in complete medium or for experimental purposes treated for $4 \mathrm{~h}$ with $10 \mu \mathrm{M}$ of CCCP (or with DMSO as a vehicle - control conditions). Proteins were extracted using Pierce ${ }^{\mathrm{TM}}$ RIPA lysis buffer (Thermo Fisher Scientific) enriched with a cocktail of protease and phosphatase inhibitors (Thermo Fisher Scientific) followed by mechanical extraction keeping lysates on ice at all times during the isolation procedure. Protein concentration was measured using the Bradford Protein Assay Kit (BIO-RAD), according to the manufacturer's instructions. $20 \mu \mathrm{g}$ of pro- 
tein lysates from each sample were loaded onto $12-15 \%$ PAGE mini gels depending on the molecular weight of the protein of interest. Proteins were transferred to the Amersham Protran $0.22 \mu \mathrm{M}$ nitrocellulose membranes (GE Healthcare Life Science). Next, membranes were blocked in 5\% non-fat dry milk in PBS for $1 \mathrm{~h}$ at room temperature, washed $5 \mathrm{~min}$ with PBS-Tween (PBST) containing 1\% Tween 20 Surfact-Amps Detergent Solution (Thermo Fisher Scientific) three times. Membranes were incubated with specific primary antibodies (dilution 1:1000 for all antibodies used): anti-TOMM20 (Thermo Fisher Scientific) and anti-Bnip3, anti-Bnip3L/Nix, antiLC3 and anti- $\beta$-actin served as an internal loading control (all from Cell Signaling Technology). Overnight incubation with primary antibodies was followed by three washing steps with PBST for 5 minutes at room temperature. Later, blots were incubated with secondary, polyclonal anti-rabbit (Sigma) antibodies (dilution 1:10000) for $1 \mathrm{~h}$ at room temperature followed by three washing steps. Blots were visualized with Clarity Western ECL Substrate solution (BIO-RAD) in FluorChem Q Image Analysis System from ProteinSimple ${ }^{\circledR}$. The level of detected proteins was quantified using ImageJ software.

Autophagosome formation. Autophagy was determined in cells cultured on $35 \mathrm{~mm}$ cell culture microDish with glass bottom (Ibidi) either in complete medium or for experimental purposes cultured for $24 \mathrm{~h}$ in medium in which glucose was replaced with $5 \mathrm{mM}$ galactose to force cells to produce ATP by oxidative phosphorylation. Autophagosome formation was evaluated using CYTO-ID ${ }^{\circledR}$ Autophagy Detection Kit (Enzo Life Sciences) by fluorescence microscopy (Olympus IX 81). CYTO-ID ${ }^{\mathbb{B}}$ Autophagy Detection Kit measures autophagic vacuoles and monitors autophagic flux in live cells using a dye that selectively labels accumulated autophagic vacuoles.

Immunofluorescence microscopy. Cybrids were grown on $0.13-0.16 \mathrm{~mm}$ sterile glass coverslips (Bionovo) in 24-well plates in complete medium. Cells were rinsed 3 times with PBS, fixed with $4 \%$ formaldehyde (Roti ${ }^{\circledR}$-Histofix 4\%; Carl Roth) for $20 \mathrm{~min}$ at room temperature, and permeabilized with $0.1 \%$ Triton (Sigma) with $3 \% \mathrm{BSA}$ for $5 \mathrm{~min}$ in $4^{\circ} \mathrm{C}$. For immunostaining glass coverslips were incubated overnight with primary antibodies diluted in PBS (1:250 for Nix and 1:800 for BNIP3). Secondary Alexa Fluor 647 dye conjugated antibodies (Thermo Fisher Scientific) were diluted to the final concentration of $4 \mu \mathrm{g} / \mathrm{ml}$, added on the coverslips and incubated at room temperature for $2 \mathrm{~h}$ in the dark. Then coverslips were rinsed 3 times with PBS, mounted
A

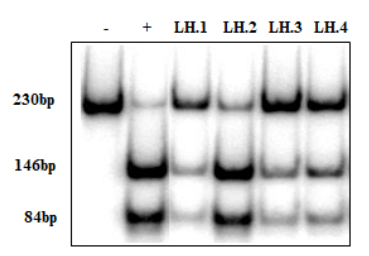

B

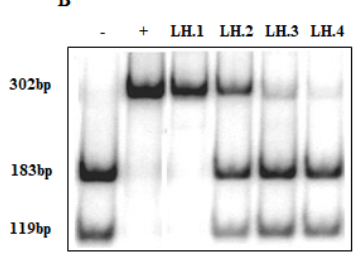

Figure 2 The mutation-specific RFLP analysis for the $11778 \mathrm{G}>\mathrm{A}$ (A) and the $3460 \mathrm{G}>\mathrm{A}$ (B) mutations in cybrid cell lines.

(A) the $11778 \mathrm{G}>\mathrm{A}$ mutation introduces a restriction site and after digestion two fragments of $146 \mathrm{bp}$ and $84 \mathrm{bp}$ were obtained. Wild type sequence is indicated by a $230 \mathrm{bp}$ band. (B) the 3460G $>$ A mutation causes the disappearance of the restriction site, and $302 \mathrm{bp}$ PCR product stays uncut. Wild type sequence is cleaved into two fragments of $183 \mathrm{bp}$ and $119 \mathrm{bp}$. LH.1-LH.4 - LHON cybrid cell lines, + positive control (with mutation), - negative control (without mutation). onto microscope slides using ProLong Gold Antifade Mountant with DAPI (Thermo Fisher Scientific) and analyzed using the Nikon A1R multiphoton confocal microscope. Microscopic pictures were analyzed using Nikon Instruments and ImageJ software.

Statistical analysis. Western blot and microscopic pictures as well as data obtained from fluorescence analyses were analyzed using ImageJ software. Protein immunoreactivity and fluorescence results were normalized to control samples, then the data was combined from 3 independent different blots/experiments. Data between two groups were compared using Student's $t$-test. The obtained differences were considered to be significant when the $p$-value was $<0.05$.

\section{RESULTS}

\section{Analysis of cybrid nuclear background and mutation heteroplasmy level}

Mutation-specific PCR-RFLP analysis of the mtDNA from cybrids indicated that in all cybrids both mutations were heteroplasmic. The degree of heteroplasmy of the $11778 \mathrm{G}>\mathrm{A}$ mutation in all cell lines exceeded 34\%, but for the $3460 \mathrm{G}>\mathrm{A}$ mutation varied from $7 \%$ to $89 \%$. The highest level of the $11778 \mathrm{G}>\mathrm{A}$ mutation was present in the LH.2cybrid cell line $(89.8 \pm 0.92 \%)$ and the highest of the $3460 \mathrm{G}>\mathrm{A}$ mutation in the LH.1 cybrid cell line $(89.3 \% \pm 0.58 \%)$. LH.1 and LH.2 cell lines were characterized by the highest level of both mutations among all the cell lines. The mutation-specific RFLP analysis for the $11778 \mathrm{G}>\mathrm{A}$ and the $3460 \mathrm{G}>\mathrm{A}$ mutations is presented in Fig. 2 and the heteroplasmy levels of each mutation for all cybrids are presented in Table 1.

Table 1. Heteroplasmy of the $11778 \mathrm{G}>\mathrm{A}$ and the $3460 \mathrm{G}>\mathrm{A}$ mutations in cybrid cell lines. LH.1-LH.4-LHON cybrid cell lines.

\begin{tabular}{lll}
\hline LHON cybrid cell line & $11778 G>8(\%)$ & $3460 G>A(\%)$ \\
\hline LH.1 & $40.7 \pm 0.68$ & $89.3 \pm 0.58$ \\
\hdashline LH. 2 & $89.8 \pm 0.92$ & $36.6 \pm 0.55$ \\
\hline LH.3 & $34.3 \pm 1.1$ & $12.7 \pm 0.9$ \\
\hdashline LH.4 & $45.4 \pm 0.75$ & $7.3 \pm 0.21$ \\
\hline
\end{tabular}

Nuclear profiles of each fibroblast and cybrid cell line as well as of $\varrho 0143 \mathrm{~B}$ were analyzed by determining 3 STR nuclear markers. The results of nuclear DNA profiling for each cell line are presented in Supplementary Material Table B (at https://ojs.ptbioch.edu.pl/index. $\mathrm{php} / \mathrm{abp} /$ ). In all derived cybrid cell lines the nuclear profile was compatible with $\varrho 0143 \mathrm{~B}$ and different from the nuclear profile of fibroblasts from which cybrids were derived indicating that selection against unfused fibroblasts and cybridisation were successful and all the cybrids had the same nuclear background of $\varrho 0$ 143B cells and were appropriate to use for experiments. For the experiments cell lines with the highest level of both LHON mutations were selected (LH.1 and LH.2).

\section{BNIP3 protein is significantly decreased in cells with an elevated level of LHON mutations}

To investigate whether LHON mutations affect the expression of proteins involved in cell death induction, we analyzed the level of BNIP3 and Nix in cybrids with close to homoplasmic either $11778 \mathrm{G}>\mathrm{A}$ (LH.2) or 


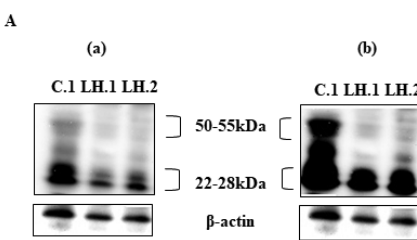

B

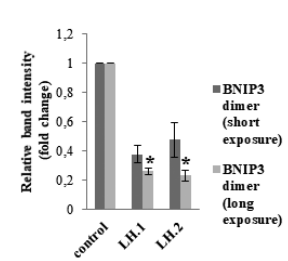

C
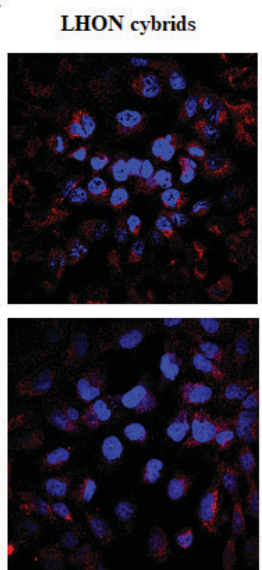

control cybrids
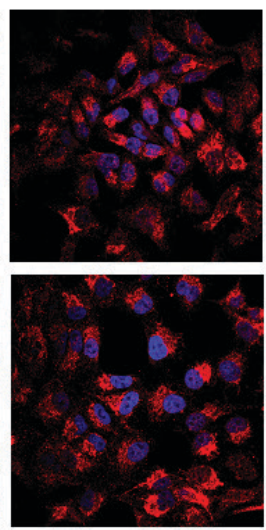

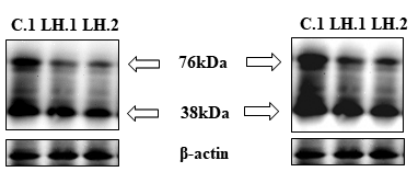

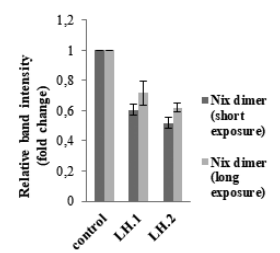

F

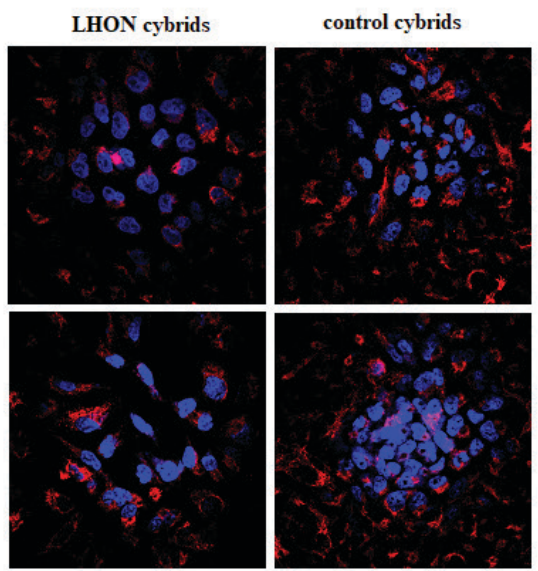

Figure 3. BNIP3 and BNIP3L/Nix level studies in LHON and control cybrids.

BNIP3 (A) and BNIP3L/Nix (D) western blotting analysis in LH.1 and LH.2 cell lines - short (a) and long (b) exposure time. Monomeric forms for BNIP3 (22-28 kDa) and for BNIP3L/Nix (38 kDa) and dimeric form for BNIP3 (50-55 kDa) and for BNIP3L/Nix (76 kDa) are present. Densitometric quantification of the immunoreactive bands for BNIP3 (B) and BNIP3L/Nix (E). The quantifications were made with the use of Image J software. Obtained values were normalized first to $\beta$-actin and the resulting values to the control. BNIP3 (C) and BNIP3L/Nix (F) analyses by confocal microscopy. Cells were grown in complete medium, fixed and immunostained with BNIP3 and BNIP3L/Nix antibodies and analyzed microscopically. BNIP3 and Nix proteins (red fluorescence), nuclei (DAPI staining; blue fluorescence), 60x magnification. Representative photographs of cells are shown. LH.1-LH.2 - LHON cybrids, C.1 - control cybrids.

3460G > A (LH.1) mutations. Western blot and confocal microscopy studies revealed that in LHON cybrids the level of BNIP3 protein was significantly decreased compared to control cells (Fig. 3). In both mutated cell lines the BNIP3 level was more than two times lower than in controls (Fig. 3A, B, C,). The Nix level in LHON cybrids was also decreased compared to controls, but the difference did not reach statistical significance (Fig. 3D, E, F).
In order to check if the different level of BNIP3 between LHON cells and controls was the consequence of different mitochondrial biogenesis, we measured mitochondrial mass in all derived cell lines. The analysis indicated that mitochondrial mass of LHON cybrids did not differ from that in controls (Fig. 4A). We also checked the level of mitochondrial protein TOMM20 in the control cell line and LH.1 and LH.2 cybrids used in the experiments and confirmed that control and LHON cell
A

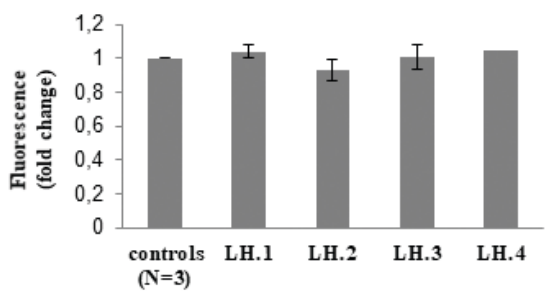

C

C.1 LH.1 LH.2

$20 \mathrm{kDa}$

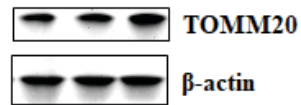

B
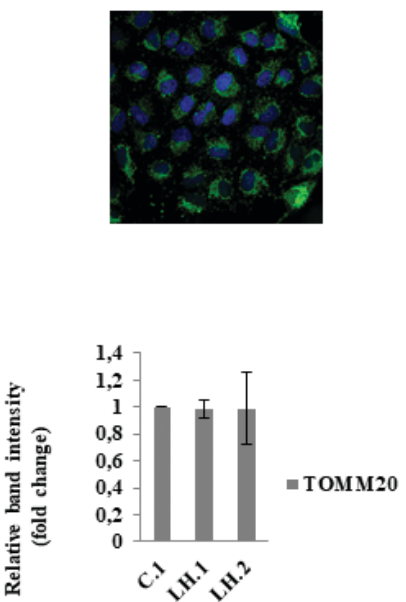

Figure 4. Mitochondrial mass analysis in cybrids by MitoTracker Green (A and B) and by western blotting (C).

(B) Representative images for MitoTracker Green fluorescence - mitochondria (green fluorescence), nuclei (DAPI staining; blue fluorescence), 60x magnification. The results of fluorescence analysis were normalized to controls and were presented as the mean standard deviation. Error bars indicate the standard deviation. LH.1-LH.4 - LHON cybrids, C.1 - control cybrids. 


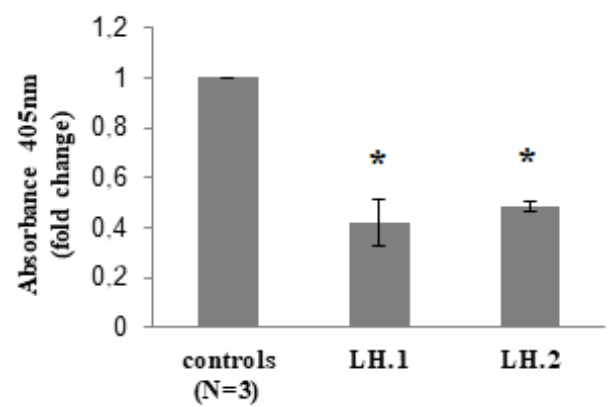

Figure 5. Analysis on free nucleosome formation in cybrids. Cells were cultured in complete medium.

Measured absorbance at $405 \mathrm{~nm}$ was normalized to control samples. Data were presented as the mean standard deviation. Error bars indicate the standard deviation. ${ }^{*} p<0.05$ comparing to controls. LH.1-LH.2 - LHON cybrids.

lines had similar levels of mitochondria (Fig. 4C). The obtained results suggest that the higher level of BNIP3 protein in controls resulted from higher expression of this protein on the mitochondrial surface of control cells rather than higher mitochondrial biogenesis.

\section{LHON cybrids with a low level of BNIP3 protein were characterized by a reduced level of autophagy and were resistant to CCCP induced cell death}

DNA fragmentation by endogenous nucleases is a key feature of apoptosis activation. Nuclear DNA is cleaved at internucleosomal linker sites resulting in formation of multiple fragments of around $180 \mathrm{bp}$, which can be detected by electrophoresis or with the use of anti-DNA and anti-histone antibodies. CCCP is a mitochondrial oxidative phosphorylation uncoupler which disrupts mitochondrial membrane potential. In the present study we wanted to check if there is a correlation between the level of BNIP3 protein and the basal level of apoptosis by measurement of mono- and oligonucleosome formation

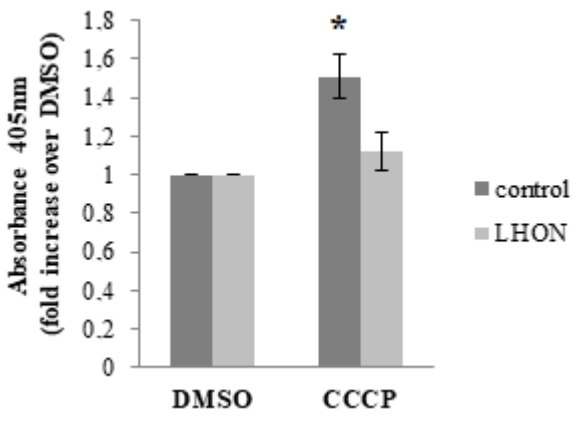

Figure 6. Analysis of CCCP-evoked apoptosis by free nucleosome detection.

Cells of controls and with LHON mutations (LH.1 cell line, marked as LHON) were cultured for $4 \mathrm{~h}$ in complete medium supplemented with DMSO (vehicle) or supplemented with $10 \mu \mathrm{M}$ CCCP. Measured absorbance at $405 \mathrm{~nm}$ was normalized to samples cultured with vehicle alone. Data are presented as the mean standard deviation. Error bars indicate the standard deviation. Data shown: ${ }^{*} p<0.05$ comparing to vehicle treated cells.

in cells cultured in complete medium. We noticed that the free nucleosome level was lower in LHON cybrids than in controls (Fig. 5). The obtained results suggest that cells with decreased BNIP3 expression compared to controls also had a lower basal level of apoptosis.

In the next stage of the experiment we wanted to compare the ability to induce apoptosis in LHON and control cybrids in stress conditions. For this purpose we cultured control and LHON cybrids with the lowest level of BNIP3 and Nix (LH.1) for $4 \mathrm{~h}$ with $10 \mu \mathrm{M}$ CCCP. The obtained results showed that control cells were more prone to CCCP-evoked apoptosis induction $(p=0.02)$ than those with LHON mutations (Fig. 6).

In the next experiments we wanted to determine autophagy in mutant and control cybrids. Cells were cultured for 24 hours in complete medium or medium in which glucose was replaced with $5 \mathrm{mM}$ galactose. We detected lower autophagosome formation in cybrids bf

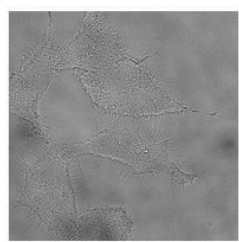

LH.1

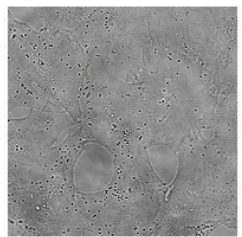

LH.2

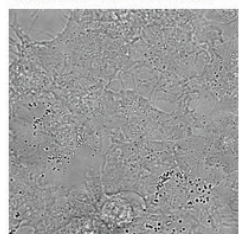

DAPI
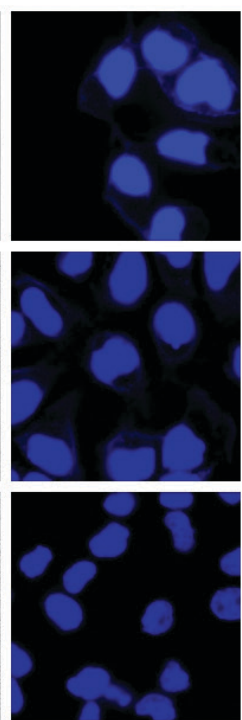

CYTO-ID

Autophagy dye
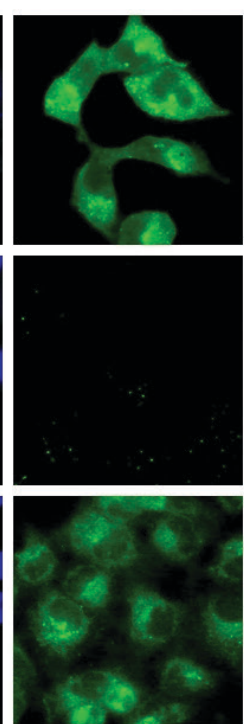
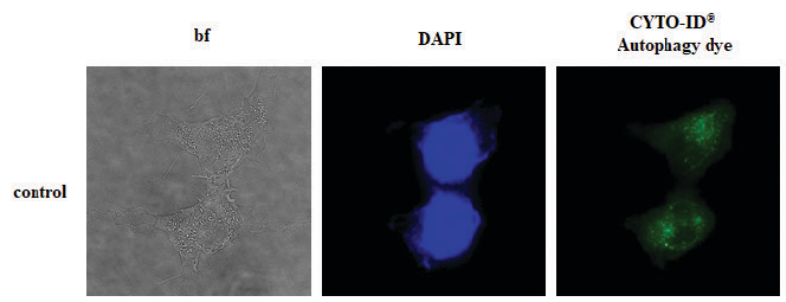

LH.1
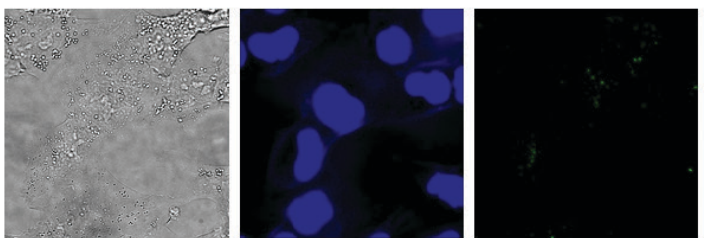

LH.2
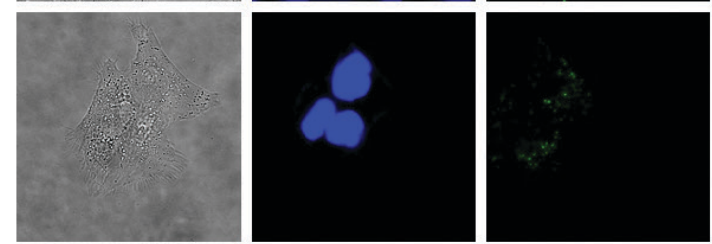

Figure 7. Autophagosomes formation analysis.

Cells were cultured for 24 hours in complete medium (A) or in medium in which glucose was replaced with 5 mM galactose and autophagosome formation with the use of fluorescence microscope was measured. Autophagosomes - green fluorescence; nuclei - DAPI staining, blue fluorescence; bf - bright field; 60x magnification 
with high level of $3460 \mathrm{G}>\mathrm{A}$ mutation (LH.1 cell line, Fig. 7A) in complete medium and in both LHON cybrids compared to control cells in galactose medium (Fig. 7B). Our findings suggest that LHON cybrid cells with lower levels of BNIP3 proteins had a lower autophagy level in stress conditions compared to controls. Autophagy involvement in LHON pathogenesis requires further investigation.

\section{DISCUSSION}

Despite numerous studies around the world, the mechanism of LHON disease is still not completely understood. We still do not know what metabolic pathway or which disturbances lead to optic nerve degeneration.

This study for the first time reports BNIP3 and Nix protein level analysis in a cybrid cell model with LHONcausing mtDNA mutations. In our study we found that BNIP3, a protein which was previously widely investigated in cancer pathogenesis, was significantly decreased in LHON cybrids compared to control cells. This proapoptotic protein was previously reported to be downregulated in pancreatic adenocarcinoma (Okami et al., 2004), myelodysplastic syndromes (Lazarini et al., 2012), acute myeloid leukemia (Lazarini et al., 2012), triple negative breast cancer (Koop et al., 2009) and colorectal cancer (He et al., 2017). Tumors which showed loss of expression of BNIP3 were found to be more prone to metastases (Koop et al., 2009). Decreased expression of BNIP3 in some cases of cancers was found to be caused by epigenetic modification, hypermethylation, of the BNIP3 promoter (Okami et al., 2004; Lazarini et al., 2012; Li et al., 2017). Treatment of BNIP3-negative pancreatic and colorectal cancer cell lines with a DNA methylation inhibitor restored BNIP3 expression and increased chemosensitivity and cell death in those cell lines (He et al., 2017; Li et al., 2017). In our studies the methylation profile was not checked and the cause of the lower level of BNIP3 in LHON cybrids requires further investigation. However, the importance of this protein in cancer pathogenesis seems to indicate that its level is important for cell function and the proper response to death stimuli, therefore it is highly probable that in neurodegenerative diseases, like LHON, it could also play an important role in cell death.

The theory, indicating elevated apoptosis as the main reason of retinal ganglion cell death in LHON, was investigated in many studies on cell lines isolated from LHON patients. It was reported that incubation of LHON cybrids harboring one of the three most frequent LHON pathogenic mutations, at positions 11778/ND4, $3460 / N D 1$, and 14484/ND6, undergo cell death when galactose replaces glucose in the culture medium, characterized by the typical hallmarks of apoptosis (Ghelli et al., 2003). Moreover, the observed cell death of LHON cells was caspase-independent (Zanna et al., 2005). Battisti and others (Battisti et al., 2004) analyzed the 2-deoxy-D-ribose induced apoptotic response of peripheral blood lymphocytes from six patients with LHON (five with the $11778 \mathrm{G}>\mathrm{A}$ and one with the $14484 \mathrm{~T}>\mathrm{C}$ mutation) and six healthy subjects. Their studies confirmed results of previous studies on LHON cybrids that cells of patients with LHON mutations had a higher rate of apoptosis than controls. Those results are in line with hypothesis that LHON mutations increase the apoptotic rate in affected patients. However, other studies of four fibroblast cell lines homoplasmic for the $11778 \mathrm{G}>\mathrm{A}$ mutation showed no significant difference in apoptotic rate between LHON cell lines compared with controls under galactose media conditions, checked by western blot analysis of cleaved caspase-3 and PARP and by TUNEL assay (Yu-Wai-Man et al., 2017). In our studies we describe a cybrid cell model with combination of two LHON mutations, which had never been analyzed previously. An interesting observation was that our LHON cybrids were resistant to apoptotic stimuli. The molecular answer for cellular stress could depend on the type of stressor as well as the cell type analyzed. In a cybrid model described by Ghelli and others (Ghelli et al., 2003) they noticed a higher level of apoptosis in cells cultured in galactose medium. Cells with defects of oxidative respiration are able to keep up the energy production when they are cultured in media with glucose, however, they show growth impairment and increased cell death when they are forced to rely on ATP synthesis by the respiratory chain in medium in which glucose is replaced with galactose. In our project we used CCCP as a stressor to provoke cell death, however, cells were cultured in high-glucose medium, and therefore cells were able to overcome energetic stress. We cannot exclude that, if we combine CCCP and galactose medium we could increase cell death also in our cell model. We also cannot exclude the possibility that prolonged cell death stimulation, when cells are beyond rescue, but apoptosis is inhibited for example because of the low level of pro-apoptotic proteins, can activate alternative cell death pathways not requiring caspase activity in LHON cells.

We can also hypothesize that the level of BNIP3 protein modifies the response to some kind of cellular stress and a decrease in the BNIP3 pro-apoptotic protein level in our LHON cell model, could negatively affect apoptosis induction, what in turn can lead to cell survival as in the case of BNIP3-negative cancer cells (He et al., 2017; Li et al., 2017). Our analyses of lymphoblasts with the $11778 \mathrm{G}>\mathrm{A}$ mutation treated with different concentrations of testosterone could support this hypothesis. We noticed that cells with the mutations expressed higher concentrations levels of both BNIP3 and BNIP3L proteins compared to controls in medium with testosterone and LHON cells were characterized by a higher level of apoptotic cell death in these conditions (not shown). These results strongly suggest that the level of BNIP3 correlates with apoptosis and cell death increases with the increasing level of this protein. Differences in expression of BNIP3 between cybrids and lymphoblasts in our studies could suggest that cell type could modify the expression of those proteins and different stressors can induce various cellular response. Moreover, the type of mutation and its level could also influence the expression of BNIP3 and BNIP3L proteins in turn modifying the reaction to stress. This could explain the different apoptosis rate between cybrids with two mutations used in our studies and those with single LHON mutations described in the literature. This issue requires further investigations. Studies on our cybrid model with double LHON mutations revealed a decrease in autophagy induction compared to controls. Reduced autophagy was previously described in cybrids with single LHON mutations (11778G $>$ A and 3460G $>$ A) (Sharma et al., 2019) and in cybrids with LHON-associated $12338 \mathrm{~T}>\mathrm{C}$ mutation in the ND5 gene (Zhang et al., 2018), which suggests that mutations responsible for LHON can negatively affect the autophagic pathway. More studies have to be conducted to check whether the BNIP3 protein level correlates with the level of autophagic cell death in cells with other mtDNA mutations. 
Additional analyses of BNIP3 and Nix levels in cells with different mtDNA mutations causing LHON and other mitochondrial diseases could help to clarify whether a reduced BNIP3 level is a common feature characteristic for mtDNA-related disorders and whether the level of BNIP3 always correlates with downregulation of apoptosis and autophagy. If a decrease of pro-apoptotic proteins like BNIP3 will be detected more often, it will confirm our observation and hypothesis that apoptosis is not the leading pathway through which cells with LHON mutations are dying.

Beyond cell death induction, recent studies suggest that BNIP3 and Nix proteins are involved in another, highly important process of mitochondrial quality control by mediation in mitochondrial repair. Miyamoto et al. (2011) described the process of intramitochondrial lysosomal degradation of oxidized proteins (MALM-Mieapinduced accumulation of lysosome-like organelles within mitochondria), in which the crucial roles are played by 3 proteins: Mieap, BNIP3 and Nix (Nakamura et al., 2017). Mieap/Nix/BNIP3 interaction at the outer mitochondrial membrane is essential for formation of pores through the double mitochondrial membrane in order to mediate the translocation of lysosomal-like structures from the cytoplasm to the mitochondrial matrix (Nakamura et al., 2017). ROS promotes p53 to activate Mieap (Mitochondria-eating protein) transcription and start the MALM pathway. Kitamura and others (Kitamura et al., 2011) indicated the importance of the Nix protein in the mitochondrial quality control system by experiments with A549 and LS174T Nix-knockdown cells. They proved that in those Nix-knockdown cell lines Mieap was mislocalized and MALM dramatically inhibited. The knockdown of endogenous BNIP3 expression in A549 cells also severely inhibited MALM (Nakamura et al., 2012), indicating that both BNIP3 and Nix are essential for proper function of the mitochondrial quality control system.

Kamino and others (Kamino et al., 2016) showed that the MALM pathway is frequently inactivated in human colorectal cancer, which in turn leads to accumulation of dysfunctional mitochondria in cells and increased reactive oxygen species generation. The accumulation of dysfunctional mitochondria in the optic nerve of LHON patients was also previously reported as well as an increased level of oxidative damage to DNA (Carelli et al., 2004; Yen et al., 2004). This can suggest that mitochondrial quality control is disturbed as in the case of MALM-deficient cancer cell lines. Those observations together with our results led us to the hypothesis that BNIP3 decrease in LHON cybrids could be a sign of a defective mitochondrial quality control system. MALM is a much longer process of protein degradation [around 24-72 h (Miyamoto et al., 2011; Nakamura et al., 2017; Nakamura et al., 2012)] than classical autophagy (a few hours). If this process is disturbed because of the low level of proteins involved in its activation it can be even extended to several days. Such a delay could result in a longer time of accumulation of defective mitochondria and deterioration of cell fitness. It is possible that a weaker but not completely inhibited mitochondrial quality control system could be involved in the pathogenesis of LHON, a disease with symptoms which appear much later in life in comparison to other mitochondrial diseases. Maintenance of a pool of healthy mitochondria is extremely important to non-dividing neuron cells, therefore the regulation of mitochondrial quality control mechanisms should be efficient to avoid accumulation of dysfunctional organelles, which could accelerate cell death. This issue is a very interesting point for the future analyses of the factors involved in LHON pathogenesis.

Rikka and others (Rikka et al., 2011) described an increase of mitochondrial protease activity and degradation of proteins involved in OXPHOS in mitochondria in mouse embryonic fibroblasts from Bax/Bak double knockout mice overexpressing BNIP3. Moreover, the silencing of BNIP3 resulted in increase of mitochondrial OXPHOS subunits which suggests that BNIP3 promoted OXPHOS protein degradation. Those observations led us to an alternative hypothesis - it is possible that a decrease in the level of BNIP3 in LHON cybrids in our study could be a defense mechanism against increased degradation of mutated subunits of complex I, which could cause faster degradation of mitochondria and accelerate ATP depletion.

Taken together, we provide the first evidence that BNIP3 protein is expressed at a lower level in cybrids with LHON-specific mutations and the decreased level of BNIP3 correlated with a higher resistance to apoptotic stimuli and decreased autophagy induction. Future investigations on other types of cellular models with different mtDNA mutations will be important to uncover the link between decreased BNIP3 levels in Leber's Hereditary Optic Neuropathy and downregulation of autophagy and resistance to apoptotic stimuli and to elucidate the cause and the consequence of the lowered expression of BNIP3 in these cells. Moreover, the level of the other Bcl-2 family pro-apoptotic and anti-apoptotic proteins should be checked in order to clarify, if BNIP3 is a critical player in cell death pathways in cells with LHON mutations.

\section{Conflict of Interest}

The authors declare no conflict of interest.

\section{Acknowledgements}

We would like to thank: Dr. Magdalena Korwin and Dr. Katarzyna Tońska for skin biopsies from LHON patients, to Dr. Aleksandra Sołyga-Z̈urek who delivered control fibroblast cell lines and to Dr. Lukasz Borowski who provided $\varrho 0143 \mathrm{~B}$ osteosarcoma cell lines for cybrid construction.

We would like to thank all the patients who agreed to participate in our study and donated skin biopsy samples used to establish fibroblast cell lines.

\section{REFERENCES}

Alston CL, Rocha MC, Lax NL, Turnbull DM, Taylor RW (2017) The genetics and pathology of mitochondrial disease. J Pathol 241: 235250. https://doi.org/10.1002/path.4809

Battisto C, Formichi P, Cardaioli E, Bianchi S, Mangiavacchi P, Tripodi SA, Tosi P (2017) Federico A. Cell response to oxidative stress induced apoptosis in patients with Leber's hereditary optic neuropathy. Mitochondrion 36: 36-42. https: //doi.org/10.1016/j. mito.2017.01.004

Carelli V, Ross-Cisneros FN, Sadun AA (2004) Mitochondrial dysfunction as a cause of optic neuropathies. Progress Retinal Eye Res 23: 53-89. https://doi.org/10.1016/j.preteyeres.2003.10.003

Ghelli A, Porcelli AM, Martinuzzi A, Carelli V, Rugolo M (2005) Caspase-independent death of Leber's hereditary optic neuropathy cybrids is driven by energetic failure and mediated by AIF and Endonuclease G. Apoptosis 10: 997-1007. https://doi.org/10.1007/ s10495-005-0742-5

Gustafsson AB (2011) Bnip3 as a Dual regulator of mitochondrial turnover and cell death in the Myocardium. Pediatric Cardiology 32: 267-274. https: //doi.org/10.1007/s00246-010-9876-5

Hamacher-Brady A, Brady NR (2016) Mitophagy programs: mechanisms and physiological implications of mitochondrial targeting by autophagy. Cell Mol Life Sci 73: 775-795. https://doi.org/10.1007/ s00018-015-2087-8 
He J, Pei L, Jiang H, Yang W, Chen J, Liang H (2017) Chemoresistance of colorectal cancer to 5-fluorouracil is associated with silencing of the BNIP3 gene through aberrant methylation. J Cancer 8: 1187-1196. https: //doi.org/10.7150/jca.18171

Imazu T, Shimizu S, Tagami S, Matsushima M, Nakamura Y, Miki T, Okuyama A, Tsuimoto Y (1999) Bcl-2/E1B 19 kDa-interacting protein 3-like protein (Bnip3L) interacts with $\mathrm{Bcl}-2 / \mathrm{Bcl}-\mathrm{xL}$ and induces apoptosis by altering mitochondrial membrane permeability. Oncogene 18: 4523-4529. https://doi.org/10.1038/sj.onc.1202722

Jankauskaite E, Bartnik E, Kodron A (2017) Investigating Leber's hereditary optic neuropathy: Cell models and future perspectives. Mitochondrion 32: 19-26. https://doi.org/10.1016/j.mito.2016.11.006

Kamino H, Nakamura Y, Tsuneki M, Sano H, Miyamoto Y, Kitamura N, Futumura M, Kanai Y, Taniguchi H, Shida D, Kanemitsu Y, Moriya Y, Yoshida K, Arakawa H (2016) Mieap-regulated mitochondrial quality control is frequently inactivated in human colorectal cancer. Oncogenesis 5: 1-11. https://doi.org/10.1038/ oncsis. 2015.43

Kitamura N, Nakamura Y, Miyamoto Y, Miyamoto T, Kabu K, Yoshida M, Futamura M, Ichionose S, Arakawa H (2011) Mieap, a p53-inducible protein, controls mitochondrial quality by repairing or eliminating unhealthy mitochondria. PLoS One 6: 1-19. https://doi. org/10.1371/journal.pone.0016060

Koop EA, van Laar T, van Wichen DF, de Weger R, van der Wall E, Diest PJ (2009) Expression of BNIP3 in invasive breast cancer: correlations with the hypoxic response and clinicopathological features. BMC Cancer 9: 1-8. https://doi.org/10.1186/1471-2407-9-175

Lazarini M, Machado-Neto J, Duarte A.S, Pericole FV, Favaro P, Barcellos KSA, Traina F, Saad STO (2012) BNIP3 is downregulated in myelodysplastic syndromes and acute myeloid leukemia and is a potential molecular target for decitabine. Blood 120: 1708

Li Y, Zhang X, Yang J, Zhang Y, Zhu D, Zhang L, Zhu Y, Li D, Zhou J (2017) Methylation of BNIP3 in pancreatic cancer inhibits the induction of mitochondrial-mediated tumor cell apoptosis. Oncotarget 8: 63208-63222. https://doi.org/10.18632/oncotarget.18736

Liu G, Zou H, Luo T, Long M, Bian J, Liu X, Gu J, Yuan Y, Song R, Wang Y, Zhu J, Liu Z (2016) Caspase-dependent and caspase-independent pathways are involved in cadmium-induced apoptosis in primary rat proximal tubular cell culture. PLoS One 11: 1-17. https://doi.org/10.1371/journal.pone.0166823

Miyamoto Y, Kitamura N, Nakamura Y, Futamura M, Miyamoto T, Yoshida M, Ono Masaya, Ichionose S, Arakawa H (2011) Possible existence of lysosome-like organella within mitochondria and its role in mitochondrial quality control. PLoS One 6: 1-20. https://doi. org/10.1371/journal.pone.0016054
Mroczek-Tońska K, Kisiel B, Piechota J, Bartnik E (2003) Leber hereditary optic neuropathy - a disease with a known molecular basis but a mysterious mechanism of pathology. J Appl Genet 44: 529-538

Nakamura Y, Arakawa H (2017) Discovery of Mieap-regulated mitochondrial quality control as a new function of tumor suppressor p53. Cancer Sci 108: 809-817. https://doi.org/10.1111/cas.13208

Nakamura Y, Kitamura N, Shinogi D, Yoshida M, Goda O, Murai R, Kamino H, Arakawa H (2012) BNIP3 and NIX mediate Mieap-induced accumulation of lysosomal proteins within mitochondria. PLoS One 7: 1-14. https://doi.org/10.1371/journal.pone.0030767

Okami J, Simeone DM, Logsdon CD (2004) Silencing of the hypoxia-inducible cell death protein BNIP3 in Pancreatic Cancer. Cancer Res 64: 5338-5346. https://doi.org/10.1158/0008-5472.CAN-04-0089

Rikka S, Quinsay M.N, Thomas R.L, Kubli D.A, Zhang X, Murphy AN, Gustafsso AB (2011) Bnip3 impairs mitochondrial bioenergetics and stimulates mitochondrial turnover. Cell Death Differentiation 18: 721-731. https://doi.org/10.1038/cdd.2010.146

Sharma LK, Tiwari M, Rai NK, Bai Y (2019) Mitophagy activation repairs Leber's hereditary optic neuropathy-associated mitochondrial dysfunction and improves cell survival. Human Mol Genet 28: $422-$ 433. https://doi.org/10.1093/hmg/ddy354

Stewart JB, Chinnery PF (2015) The dynamics of mitochondrial DNA heteroplasmy: implications for human health and disease. Nature Rev 16: 530-542. https://doi.org/10.1038/nrg3966

Tońska K, Kurzawa M, Ambroziak AM, Korwin-Rujna M, Szaflik P, Grabowska E, Szaflik J, Bartnik E (2008) A family with 3460G>A and $11778 \mathrm{G}>\mathrm{A}$ mutations and haplogroup analysis of Polish Leber hereditary optic neuropathy patients. Mitochondrion 8: 383-388. https://doi.org/10.1016/j.mito.2008.08.002

Yen M.Y, Kao S.H, Wang A.G, Wei YH (2004) Increased 8-hydroxy-2'-deoxyguanosine in leukocyte DNA in Leber's hereditary optic neuropath. Biochem Mol Biol 45: 1688-1691. https://doi. org/10.1167/iovs.03-0568

Yu-Wai-Man P, Soiferman D, Moore DG, Burte F, Saada A (2017) Evaluating the therapeutic potential of idebenone and related quinine analogues in Leber hereditary optic neuropathy. Mitochondrion 36: 36-42. https://doi.org/10.1016/j.mito.2017.01.004

Zanna C, Ghelli A, Porcelli A.M, Carelli V, Martinuzzi A, Rugolo M (2003) Apoptotic cell death of cybrid cells bearing Leber's hereditary optic neuropathy mutations is caspase independent. Ann New York Acad Sci 1010: 213-217. https://doi.org/10.1196/annals.1299.037

Zhang J, Ji Y, Lu Y, Fu R, Xu M, Liu X, Guan MX (2018) Leber's hereditary optic neuropathy (LHON)-associated ND5 12338T $>$ C mutation altered the assembly and function of complex I, apoptosis and mitophagy. Human Mol Genet 27: 1999-2011. https://doi. org $/ 10.1093 / \mathrm{hmg} /$ ddy107 